\title{
CORRIGENDUM
}

\section{Cerebellar Purkinje cell p75 neurotrophin receptor and autistic behavior}

LT Lotta, K Conrad, D Cory-Slechta and NF Schor

Translational Psychiatry (2014) 4, e476; doi:10.1038/tp.2014.118; published online 28 October 2014

Correction to: Translational Psychiatry (2014) 4, e416; doi:10.1038/ tp.2014.55; published online 29 July 2014

Following publication, the authors were informed that the strain name of the mice used was incorrect. The error occurred in the first paragraph of the Materials and Methods section. The correct mouse strain is: B6.Cg-Tg(Pcp2-cre)3555Jdhu/J (mouse) Stock Number: 010536.

Also in the first paragraph of the Materials and Methods section, the URL containing further information about the mouse strain should be http://jaxmice.jax.org/strain/010536.html 